\section{F-fluorodeoxyglucose positron-emission tomography/CT and lung involvement in systemic sclerosis}

Systemic sclerosis (SSc) early lung involvement (interstitial lung disease (ILD)) is characterised by ground glass opacities (GGO) at high-resolution CT (HRCT). ${ }^{1} 2$ The literature provides conflicting interpretations of GGO's clinical significance, ${ }^{3-5}$ and whether it represents inflammation or early fibrotic changes is a dilemma. In fact, HRCT cannot discriminate between 'active inflammatory' and 'established fibrotic' GGO. ${ }^{6}$ Instead, 18-F fluoro-deoxy-d-glucose positron-emission tomography/ CT (18F-FDG-PET/CT) locates areas of increased metabolic activity, ${ }^{78}$ but no data on the 'established fibrotic' GGO metabolic activity has been demonstrated yet. We aimed at evaluating if 18F-FDG-PET/CT scan may identify GGO inflammatory component in SSc-ILD. ${ }^{9}$

Seven patients with SSc (six females; mean age 59.56 \pm 9.15 years, median disease duration 5 years) from the Rheumatology Outpatient Clinic, University of Florence underwent a 18F-FDG-PET/CT to rule out the presence of a neoplasia for a lung nodule detected at chest HRCT. HRCT pulmonary segments were classified as 'negative' (normal morphology) and 'positive' (presence of GGO), and the Warrick score was used to quantify ILD at HRCT. ${ }^{10}$ 18F-FDG-PET/CT images were retrospectively analysed by two independent blinded nuclear medicine physicians, obtaining mean standardised uptake value (mSUV) for regions-of-interest for each lung segment, using standard PET/CT scanner workstation tools. These values were normalised (nmSUV) by comparison with sex, age, height and weight-matched controls selected from a database of subjects with negative PET/CT scans and no thoracic and systemic diseases (reference value of 1). Clinical features (including smoking exposure), laboratory workup, chest echocardiography and pulmonary function tests were recorded.

All patients with SSc were non-smokers, and cancer (as assessed by PET/CT and follow-up HRCTs until August 2017), cardiac function abnormalities or increased systolic pulmonary artery pressure were ruled out.

In the four patients with a Warrick score $=0$, the lung segments were all 'negative' (group A) and had an nmSUV (mean 0.97; $95 \%$ CI 0.93 to $1.01, \mathrm{p}=0.14$ ) which was not different to reference value of 1 . In the three patients with Warrick score $>0$, a significantly higher nmSUV than reference value of 1 was detected both for 'positive' (group C, mean 1.53; CI 1.42 to $1.65, \mathrm{p}<0.0001$ ) and for 'negative' (group B mean 1.29; CI 1.22 to $1.37, \mathrm{p}<0.0001)$ lung segments. Group $\mathrm{C}$ showed a $24 \%$ higher nmSUV than group B (CI 0.13 to $0.33, \mathrm{p}<00$ 001) (figure 1). Interestingly, lung segments of group B had a $32 \%$ higher $18 \mathrm{~F}-\mathrm{FDG}$ uptake than in group A (CI 0.17 to 0.48 , $\mathrm{p}<0.0001)$.

This preliminary study showed that morphologically 'positive' GGO segments present a clear 18F-FDG uptake, suggesting the existence of an increased metabolic activity of GGO. This 18-FDG uptake could be potentially due to inflammation but could also be seen as a reflection of a concomitant active phenomenon due to underlying pathogenetic mechanisms (ie, high fibroblast activity). ${ }^{11}$

Although these data indicate that PET/CT may disclose an underlying inflammatory process not yet evidenced by HRCT, still it remains to be determined whether early parenchymal involvement may be identified before this is evident at HRCT. In fact, the metabolic activity observed in group B could be related to a lung SSc-related 'impairment' not detectable at HRCT, possibly representing a lung reaction to the disease, stronger in those subjects with some segments affected by GGO. In the

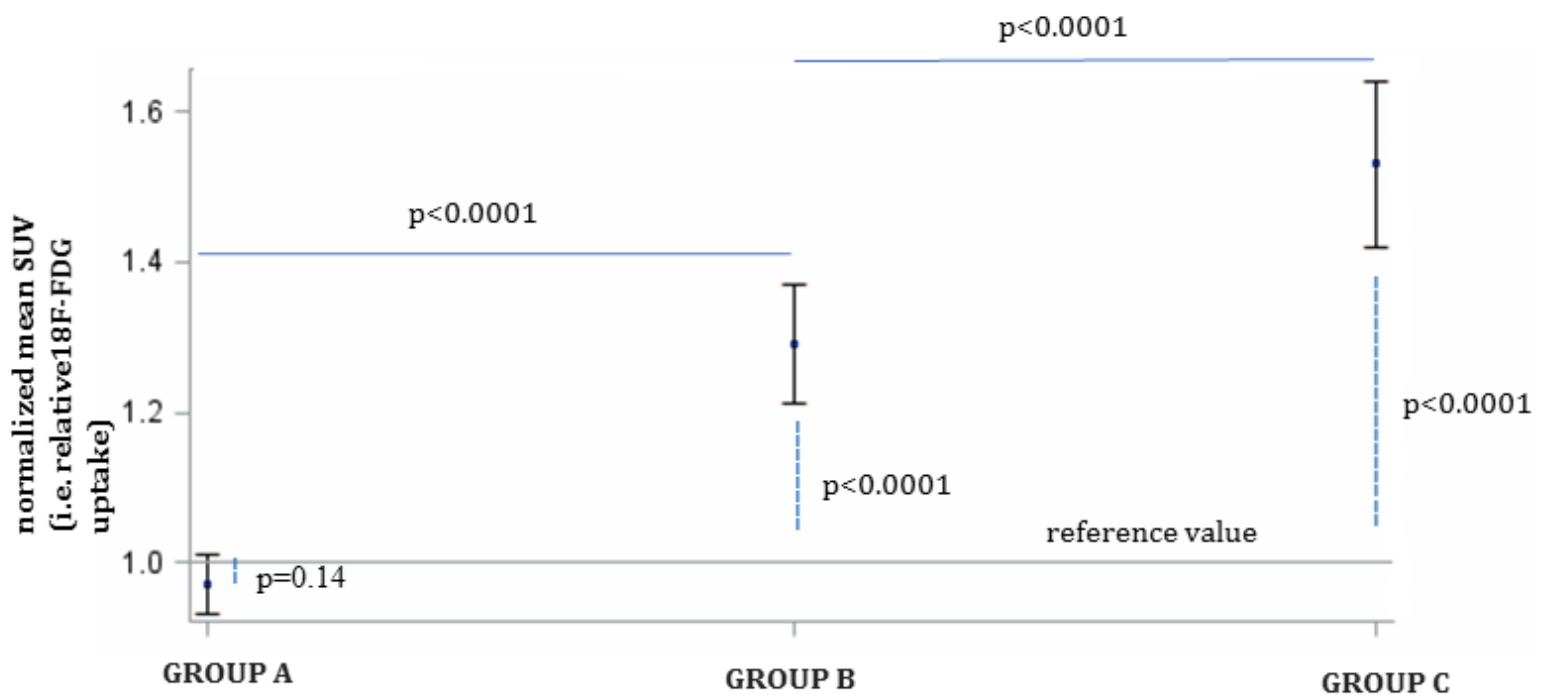

\author{
"negative" segments of \\ Warrick score $=0 \mathrm{SSc}$ patients
}

\author{
"negative" segments of \\ Warrick score $>0$ SSc patients
}

\section{"positive" segments of Warrick score $>0 \mathrm{SSc}$ patients}

Figure 1 Differences in 18F-FDG uptake between 'positive' segments of Warrick score $>0$ SSc patients, 'negative' segments of Warrick score $>0$ SSc patients and 'negative' segments of Warrick score $=0$ SSc patients versus attended normalised control value (=1). 18F-FDG, 18-F fluoro-deoxy-dglucose; SSc, systemic sclerosis; SUV, standardised uptake value. 
future, the evolution of this specific metabolic activity could be verified with an HRCT follow-up. The fact that 18F-FDG PET/ CT might be useful to evaluate the response to treatment during follow-up of patients with SSc, as already used in oncology, remains a working hypothesis because the radiation load is a serious issue which could limit this approach. Further studies on a larger population are warranted to verify our data, preliminary results and possibly provide a prognostic significance of PET/CT positivity in patients with SSc.

\section{Silvia Bellando-Randone, ${ }^{1,2}$ Luca Tartarelli, ${ }^{3}$ Edorardo Cavigli, ${ }^{4}$ Lorenzo Tofani, ${ }^{5}$ Cosimo Bruni, ${ }^{1,2}$ Gemma Lepri, ${ }^{1,2}$ Jelena Blagojevic, ${ }^{1,2}$ Alberto Moggi-Pignone, ${ }^{6}$ Carina Mihai, ${ }^{7}$ Jerome Avouac, ${ }^{8}$ Alessandro Passeri, ${ }^{3}$ Maria Teresa De Cristofaro, ${ }^{3}$ Oliver Distler, ${ }^{7}$ Yannick Allanore, ${ }^{8}$ Serena Guiducci, ${ }^{1,2}$ Marco Matucci-Cerinic ${ }^{1,2}$}

${ }^{1}$ Department of Experimental and Clinical Medicine, University of Florence, Florence, Italy

${ }^{2}$ Division of Rheumatology AOUC, Department of Geriatric Medicine, University of Florence, Florence, Italy

"Department of Biomedical, Experimental and Clinical Sciences, "Mario Serio", Nuclear Medicine Unit, University of Florence, Florence, Italy

${ }^{4}$ Department of Radiodiagnostic and Emergency, Careggi University Hospital, Florence, Italy

${ }^{5}$ Department of Neurosciences, Psychology, Drug Research and Child Health, University of Florence, Florence, Italy

${ }^{6}$ Internal Medicine of Careggi University Hospital, Florence, Italy

${ }^{7}$ Department of Rheumatology, University Hospital Zurich, Zurich, Switzerland

${ }^{8}$ Department of Rheumatology A, Cochin Hospital, INSERM U1016, Paris Descartes University, Paris, France

Correspondence to Silvia Bellando-Randone, Department of Experimental and Clinical Medicine, University of Florence, Florence 50139, Italy;

s.bellandorandone@gmail.com

\section{Handling editor Josef S Smolen}

Contributors SB-R and LTa: contribution to conception and design of the study, interpretation of data, drafting the manuscript and final approval. EC: execution, analysis and interpretation of data, final approval. LTo: analysis and interpretation of data and final approval. CB: drafting the manuscript and final approval. GL, JB, CM, JA and SG: revising the manuscript critically and final approval. AM-P: contribution to conception and design of the study and final approval. AP and MTDC: execution, interpretation of data and final approval. OD and YA: contribution to conception and the design of the study, revising the manuscript critically and final approval. MM-C: contribution to conception and the design of the study, interpretation of data, revising the manuscript critically and final approval.

Funding The authors have not declared a specific grant for this research from any funding agency in the public, commercial or not-for-profit sectors.

Competing interests None declared.
Patient consent Obtained.

Ethics approval As case series, ethics committee approval was not required in accordance with the policy of our institution.

Provenance and peer review Not commissioned; externally peer reviewed. (C) Author(s) (or their employer(s)) 2018. No commercial re-use. See rights and permissions. Published by BMJ.

This work has been previously presented at a conference and published as a conference abstract in 2018.

$$
\text { Check for updates }
$$

To cite Bellando-Randone S, Tartarelli L, Cavigli E, et al. Ann Rheum Dis Epub ahead of print: [please include Day Month Year]. doi:10.1136/annrheumdis-2018-213376

Received 7 March 2018

Revised 1 October 2018

Accepted 2 October 2018

Ann Rheum Dis 2018:0:1-2. doi:10.1136/annrheumdis-2018-213376

\section{REFERENCES}

1 Volkmann ER, Chung A, Tashkin DP. Managing systemic sclerosis-related interstitial Lung Disease in the modern treatment era. J Scleroderma Relat Disord 2017;2:72-83.

2 Jaeger VK, Wirz EG, Allanore Y, et al. Incidences and risk factors of organ manifestations in the early course of systemic sclerosis: a longitudinal eustar study. PLoS One 2016;11:e0163894.

3 Shah RM, Jimenez S, Wechsler R. Significance of ground-glass opacity on HRCT in long-term follow-up of patients with systemic sclerosis. J Thorac Imaging 2007:22:120-4.

4 Remy-Jardin M, Giraud F, Remy J, et al. Importance of ground-glass attenuation in chronic diffuse infiltrative lung disease: pathologic-CT correlation. Radiology 1993; 189:693-8

5 Latsi PI, Wells AU. Evaluation and management of alveolitis and interstitial lung disease in scleroderma. Curr Opin Rheumatol 2003;15:748-55.

6 Launay D, Remy-Jardin M, Michon-Pasturel U, et al. High resolution computed tomography in fibrosing alveolitis associated with systemic sclerosis. J Rheumatol 2006:33:1789-801.

7 Kinahan PE, Fletcher JW. PET/CT Standardized Uptake Values (SUVs) in clinical practice and assessing response to therapy. Semin Ultrasound CT MR 2010:31:496-505

8 Uehara T, Takeno M, Hama M, et al. Deep-inspiration breath-hold 18F-FDG-PET/CT is useful for assessment of connective tissue disease associated interstitial pneumonia. Mod Rheumatol 2016;26:121-7.

9 Bellando Randone S, Tartarelli L, Cavigli E. 18F-Fluorodeoxyglucose (FDG) Positron Emission Tomography/ Computer Tomography (PET/CT) Scan and Lung Involvement in Systemic Sclerosis (SSC): which is the Future? I Scleroderma Rel Disord 2018;3:168.

10 Warrick JH, Bhalla M, Schabel SI, et al. High resolution computed tomography in early scleroderma lung disease. J Rheumatol 1991;18:1520-8.

11 Varga J, Trojanowska M, Kuwana M. Pathogenesis of systemic sclerosis: recent insights of molecular and cellular mechanisms and therapeutic opportunities. J Scleroderma Relat Disord 2017:2:137-52. 